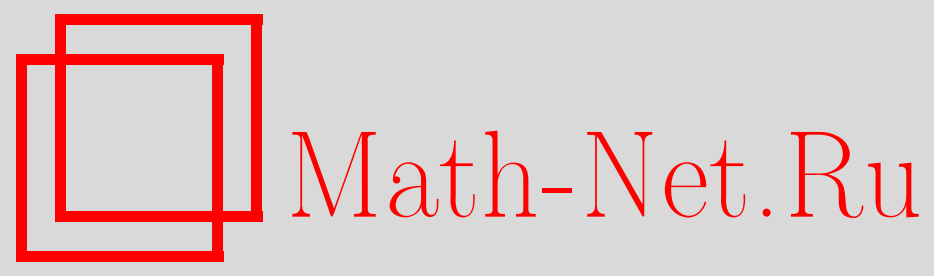

В. А. Воблый, Некоторые необходимые условия хроматичности многочлена, Дискрет. матем., 2001, том 13, выпуск 1, 73-77

DOI: https://doi.org/10.4213/dm278

Использование Общероссийского математического портала Math-Net.Ru подразумевает, что вы прочитали и согласны с пользовательским соглашением http://www . mathnet.ru/rus/agreement

Параметры загрузки:

IP: 54.209 .52 .79

26 апреля 2023 г., 12:46:37 
УДК 519.1

\title{
Некоторые необходимые условия хроматичности многочлена
}

\author{
(c) 2001 г. В. А. Воблый
}

Предложены два необходимых условия хроматичности многочлена, которые в ряде случаев оказываются сильнее некоторых известных условий.

Пусть $P(G, \lambda)$ - хроматический многочлен графа $G$ с $p$ помеченными вершинами. Известно (см. [1-5]) много необходимых условий того, что многочлен $P(\lambda)$ степени $p$ с целыми коэффициентами является хроматическим многочленов некоторого графа $G$. В этой статье предложены еще два необходимых условия хроматичности многочлена, которые в ряде случаев оказываются сильнее некоторых известных условий.

Теорема 1. Пусть

$$
p(G, \lambda)=\sum_{k=0}^{p} a_{k} \lambda^{k}
$$

- хроматический многочлен связного графа $g$ с р вершинами. Тогда для любого $j=1, \ldots, p$

$$
(-1)^{p-j} \sum_{k=j}^{p}\left(\begin{array}{l}
k-1 \\
j-1
\end{array}\right) a_{k} \geqslant 0 .
$$

Доказательство. Известно [6], что в разложении

$$
P(G, \lambda)=\sum_{k=1}^{p} P\left(T_{k}, \lambda\right)
$$

по базису из деревьев $T_{k} \mathrm{c} k$ вершинами коэффициенты $b_{k}$ чередуются по знаку, то есть $b_{k}=(-1)^{p-k}\left|b_{k}\right|, k=1, \ldots, p$. Подставляя в (2) известное выражение для хроматического многочлена дерева

$$
P\left(T_{k}, \lambda\right)=\lambda(1-\lambda)^{k-1}
$$

(см. [1]), после замены переменной $\lambda=\mu+1$, получим, что

$$
P(G, \mu+1)=\sum_{k=0}^{p} a_{k}(\mu+1)^{k}=\sum_{k=1}^{p} b_{k}(\mu+1)^{k-1} .
$$


Так как $a_{0}=0$, деля обе части (3) на $\mu+1$, применяя формулу бинома Ньютона и изменяя порядок суммирования, находим, что

$$
\begin{aligned}
\sum_{k=1}^{p} b_{k} \mu^{k-1} & =\sum_{k=1}^{p} a_{k}(\mu+1)^{k-1}=\sum_{k=1}^{p} \sum_{i=0}^{k-1}\left(\begin{array}{c}
k-1 \\
i
\end{array}\right) \mu^{i} a_{k} \\
& =\sum_{k=1}^{p} \sum_{j=1}^{k}\left(\begin{array}{c}
k-1 \\
j-1
\end{array}\right) \mu^{j-1} a_{k}=\sum_{j=1}^{p} \sum_{k=j}^{p}\left(\begin{array}{c}
k-1 \\
j-1
\end{array}\right) a_{k} \mu^{j-1}
\end{aligned}
$$

Приравнивая коэффициенты при одинаковых степенях $\mu$, находим, что

$$
b_{j}=\sum_{k=j}^{p}\left(\begin{array}{c}
k-1 \\
j-1
\end{array}\right) a_{k}=(-1)^{p-j}\left|b_{j}\right| .
$$

Следовательно, для любого $j=1, \ldots, p$

$$
\left|b_{j}\right|=(-1)^{p-j} \sum_{k=j}^{p}\left(\begin{array}{l}
k-1 \\
j-1
\end{array}\right) a_{k} \geqslant 0 .
$$

Отметим, что для $j=p$ неравенство (1) равносильно тому, что $a_{p} \geqslant 0$, а для $j=p-1$ оно равносильно неравенству $a_{p-1}+(p-1) a_{p} \leqslant 0$, или $-q+p-1 \leqslant 0$, то есть условию связности графа. При $j=1$ условие (1) превращается в неравенство

$$
(-1)^{p-1} \sum_{k=1}^{p} a_{k} \geqslant 0
$$

которое следует из условия Оре (см. [2])

$$
P(G, 1)=\sum_{k=1}^{p} a_{k}=0 .
$$

Таким образом, имеет смысл использовать (1) при $j=2, \ldots, p-2$.

Теорема 2. Пусть $P(G, \lambda)$ - хроматический многочлен графа $G$ с $p$ вершинами. Тогда для любого $j=2, \ldots, p$

$$
P(G, \lambda) \geqslant \sum_{s=1}^{j-1}(-1)^{j-s+1}\left(\begin{array}{c}
p+1 \\
j-s
\end{array}\right) P(G, s) .
$$

Доказательство. В [7] приведено тождество

$$
\sum_{n=0}^{\infty} P(G, n) x^{n}=\frac{1}{(1-x)^{p+1}} \sum_{i=0}^{p} q_{i} x^{i}
$$

где $q_{i} \geqslant 0$ для любого $i=0,1, \ldots, p$. Умножим обе части равенства (5) на множитель $(1-x)^{p+1}$, затем разложим его по формуле бинома Ньютона и получим равенство

$$
\sum_{m=0}^{p+1}(-1)^{m}\left(\begin{array}{c}
p+1 \\
m
\end{array}\right) x^{m} \sum_{n=1}^{\infty} P(G, n) x^{n}=\sum_{i=0}^{p} q_{i} x^{i} .
$$


Используя формулу для общего члена произведения степенных рядов

$$
\sum_{j=0}^{\infty} c_{j} x^{j} \sum_{j=0}^{\infty} d_{j} x^{j}=\sum_{j=0}^{\infty} \sum_{s=0}^{j} c_{s} d_{j-s} x^{j}
$$

где

$$
c_{j}=P(G, j), \quad d_{j}=(-1)^{j}\left(\begin{array}{c}
p+1 \\
j
\end{array}\right),
$$

получим, что для любого $j=0,1, \ldots, p$

$$
q_{j}=\sum_{s=0}^{j}(-1)^{j-s}\left(\begin{array}{l}
p+1 \\
j-s
\end{array}\right) P(G, s) \geqslant 0 .
$$

Выделяя слагаемое, соответствующее $s=j$, и перенеся оставшуюся часть суммы в правую часть неравенства, завершаем доказательство.

Интересно сравнить (1) и (4) с необходимыми условиями Котляра [5] хроматичности многочлена:

$$
\sum_{k=j}^{p} S(k, j) a_{k} \geqslant 0, \quad j-1, \ldots, p
$$

где $S(k, j)$ - числа Стирлинга 2-го рода, и необходимыми условиями Хватала [4]:

$$
\Delta^{j+1} P(G, 0) \geqslant \frac{J}{j-1}\left(2^{p / j}-2\right) \Delta^{j} P(G, 0), \quad j=2, \ldots, p-1 .
$$

Многочлен

$$
P(\lambda)=\lambda^{5}-4 \lambda^{4}+7 \lambda^{3}-5 \lambda^{2}+\lambda
$$

не является хроматическим, так как он должен был бы соответствовать графу с 5 вершинами, 4 ребрами и 1 компонентой связности, то есть дереву $T_{5}$. Однако

$$
P\left(T_{5}, \lambda\right)=\lambda^{5}-4 \lambda^{4}+6 \lambda^{3}-4 \lambda^{2}+\lambda .
$$

Условие (1) не выполнено при $j=2$, хотя условия (4), (6) и (7) выполняются при любом допустимом значении $j$. В то же время для нехроматического многочлена

$$
P(\lambda)=\lambda^{4}-3 \lambda^{3}+6 \lambda^{2}-4 \lambda,
$$

соответствующего графу с 4 вершинами, 3 ребрами и 1 компонентой связности и отличающегося от

$$
P\left(T_{4}, \lambda\right)=\lambda^{4}-3 \lambda^{3}+\lambda^{2}-\lambda,
$$

условия (1), (6) и (7) выполнены, а условие (4) не выполняется при $j=3$.

Оказывается, что условие (4) всегда сильнее условия Котляра.

Теорема 3. Необходимое условие Котляра (6) хроматичности многочлена следует из условия (4). 
Доказательство. Действительно, подставляя в левую часть неравенства (6) выражение для $a_{k}$ через $P(G, \lambda)$, получаем, что

$$
\begin{aligned}
\gamma_{j} & =\sum_{k=j}^{p} S(k, j) a_{k} \\
& =\sum_{k=j}^{p} S(k, j) \operatorname{Coef}_{\lambda} P(G, \lambda) \lambda^{-k-1} .
\end{aligned}
$$

Переставляя знаки операций суммирования и взятия коэффициента при степени многочлена и используя производящую функцию чисел Стирлинга 2-го рода (см. например [8])

$$
\sum_{k=j}^{\infty} S(k, j) t^{k}=\frac{1}{j !} \sum_{i=0}^{j}(-1)^{j-i}\left(\begin{array}{l}
j \\
i
\end{array}\right) \frac{1}{1-i t}
$$

находим, что

$$
\begin{aligned}
\gamma_{j} & =\operatorname{Coef}_{\lambda} \lambda^{-1} P(G, \lambda) \sum_{k=j}^{\infty} S(k, j) \lambda^{-k} \\
& =\frac{1}{j !} \sum_{i=0}^{j}(-1)^{j-i}\left(\begin{array}{l}
j \\
i
\end{array}\right) \operatorname{Coef}_{\lambda} \frac{P(G, \lambda)}{\lambda-i} \\
& =\frac{1}{j !} \sum_{i=0}^{j}(-1)^{j-i}\left(\begin{array}{l}
j \\
i
\end{array}\right) P(G, i) \\
& =\frac{1}{j !} \Delta^{j} P(G, 0) .
\end{aligned}
$$

Следовательно, условие Котляра (6) равносильно условию

$$
\Delta^{j} P(G, 0) \geqslant 0, \quad j=1, \ldots, p .
$$

Подставляя в равенство (5) выражение $P(G, n)$ по формуле Ньютона

$$
P(G, n)=\sum_{j=0}^{n}\left(\begin{array}{l}
n \\
j
\end{array}\right) \Delta^{j} P(G, 0)
$$

и изменяя порядок суммирования, получаем, что

$$
\begin{aligned}
\sum_{i=0}^{p} q_{i} x^{i} & =(1-x)^{p+1} \sum_{n=0}^{\infty} \sum_{j=0}^{n}\left(\begin{array}{l}
n \\
j
\end{array}\right) \Delta^{j} P(G, 0) x^{n} \\
& =(1-x)^{p+1} \sum_{j=0}^{\infty} \Delta^{j} P(G, 0) \sum_{n=j}^{\infty}\left(\begin{array}{l}
n \\
j
\end{array}\right) x^{n}
\end{aligned}
$$

Делая замену индекса суммирования $i=n-j$ и используя ряд (см., например, [9])

$$
\sum_{i=0}^{\infty} \frac{(i+j) !}{i !} x^{i}=\frac{j !}{(1-x)^{j+1}}
$$


находим, что

$$
\sum_{i=0}^{p} q_{i} x^{i}=(1-x)^{p} \sum_{j=0}^{\infty} \Delta^{j} P(G, 0)\left(\frac{x}{1-x}\right)^{j} .
$$

После замены переменной $t=x /(1-x)$ и применения бинома Ньютона получим равенства

$$
\begin{aligned}
\sum_{j=0}^{\infty} \Delta^{j} P(G, 0) t^{j} & =\sum_{i=0}^{p} q_{i} t^{i}(t+1)^{p+1-i} \\
& =\sum_{i=0}^{p} \sum_{s=0}^{p+1-i}\left(\begin{array}{c}
p+1-i \\
j-i
\end{array}\right) q_{i} t^{i+s}
\end{aligned}
$$

Наконец, приравнивая коэффициенты при одинаковых степенях $t$, находим, что

$$
\Delta^{j} P(G, 0)=\sum_{i=0}^{p}\left(\begin{array}{c}
p+1-i \\
j-i
\end{array}\right) q_{i}=\sum_{i=0}^{j}\left(\begin{array}{c}
p+1-i \\
j-i
\end{array}\right) q_{i} \geqslant 0
$$

при $q_{i} \geqslant 0$, и таким образом завершим доказательство.

Отметим, что легко доказывается по индукции, что условие Котляра является следствием также условием Хватала.

\section{Список литературы}

1. Харари Ф., Теория графов. Мир, Москва, 1973.

2. Оре О., Теория графов. Наука, Москва, 1968.

3. Read R. C., An introduction to chromatic polynomials. J. Combin. Theory (1968) 4, 52-71.

4. Wolf H. S., Which polynomials are chromatic? Colloq. Intern. sulle Theorie Combinatorie, Rome, 1976, 247-256.

5. Котляр Б. Д., Об одном необходимом условии хроматичности многочлена. Кибернетика и системный анализ (1998) №5, 176-178.

6. Adam A. A., Broere J., Chromatic polynomials of graphs in terms of trees. J. Math. Phys. Sci. (1993) 27, 231-240.

7. Linial N., Craph coloring and monotone functions on posets. Discrete Math. (1986) 58, №1, 97-98.

8. Риордан Дж., Введение в хомбинаторный анализ. ИЛ, Москва, 1963.

9. Прудников Ф. П., Интеграль и рлды. Наука, Москва, 1981.

Статья поступила 15.09.1999. 